\title{
Connections between steel and aluminium using adhesive bonding com- bined with self-piercing riveting
}

\author{
Matthias Reil ${ }^{1,2,3, *}$, David Morin ${ }^{2,3}$, Magnus Langseth ${ }^{2,3}$, and Octavian Knoll ${ }^{1}$ \\ ${ }^{1}$ BMW Group, Knorrstrasse 147, 80937 Munich, Germany \\ ${ }^{2}$ Centre for Advanced Structural Analysis (CASA), NTNU, NO-7491 Trondheim, Norway \\ ${ }^{3}$ Structural Impact Laboratory (SIMLab), Department of Structural Engineering, NTNU, NO-7491 Trondheim, Norway
}

\begin{abstract}
The multi-material design of modern car bodies requires joining technologies for dissimilar materials. Adhesive bonding in combination with self-piercing riveting is widely used for joining steel and aluminium structures. To guarantee crashworthiness and reliability of a car body, accurate and efficient numerical models of its materials and connections are required. Suitable component test setups are necessary for development and validation of such models. In this work, a novel test setup for adhesively bonded and point-wise connected components is presented. Here, load combinations comparable to a vehicle crash are introduced into the connections. The developed setup facilitates successive failure of multiple connections and enables a broad validation of numerical connection models.
\end{abstract}

\section{Introduction}

Stricter legal and social requirements on pollutant emissions and energy consumption of passenger cars increase the demand for lightweight vehicle structures. Therefore, a multi-material design approach becomes essential in the design of a modern car body. However, joining of dissimilar materials is a challenging task and requires the application of new joining technologies. Self-Piercing Riveting (SPR) in combination with adhesive bonding is widely used in modern car bodies for joining steel and aluminium structures. Virtual tests using numerical methods are applied to improve the crashworthiness of vehicle structures and ensure passenger safety. Consequently, accurate and efficient numerical models of the applied joining technologies are required. Tests on component level are important for development and validation of such models. Here, loading conditions comparable to a vehicle crash are introduced while cost and complexity are reduced significantly. The literature provides information on component tests of adhesively bonded connections $[1,2]$ as well as SPR connections [3, 4]. However, both joining technologies are combined in steel-aluminium vehicle structures. Therefore, a component test setup for this hybrid joining technology is required.

In this study, a novel component test for joined steelaluminium structures is presented. Here, two Hat Profile (HP) sections are joined using adhesive bonding combined with SPR and tested in a 3-point bending setup (Figure 1). The SPR process requires access to the joining location from both sides which is enabled by the open geometry of the HP sections. The well-defined boundary conditions of the 3-point bending setup ensure a simple correlation with

\footnotetext{
*e-mail: matthias.re.reil@bmw.de
}

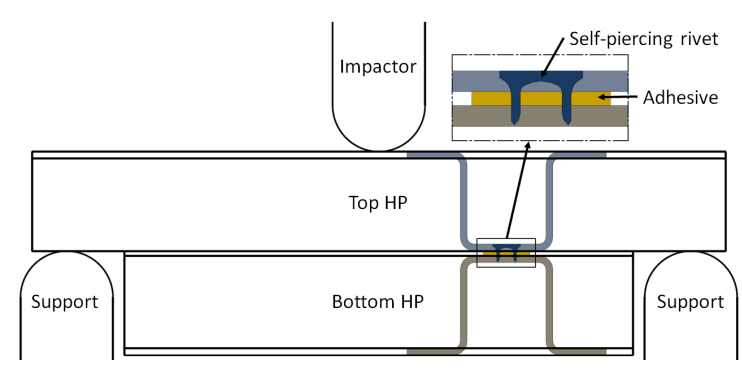

Fig. 1. Principle of the new component test setup for hybrid connections.

numerical models. A steel-aluminium material combination representative for modern car bodies was chosen. The top HP section is bent from sheets of cold rolled microalloyed steel with a nominal thickness of $1.2 \mathrm{~mm}$. The bottom HP section is bent from 6000 series alloy aluminium sheets with a nominal thickness of $2.5 \mathrm{~mm}$. The HP sections are joined using the crash modified, hot curing epoxy adhesive SikaPower SP498 and self-piercing rivets of type Böllhoff RIVSET 5.3x5 SKR. The adhesive layer has a nominal width of $15 \mathrm{~mm}$ and a nominal thickness of 0.3 $\mathrm{mm}$. Ten SPR connections are distributed evenly over the length of the lower HP section.

\section{Numerical design optimization}

\subsection{Numerical model}

Finite Element (FE) simulations were performed to optimize the component test setup. The commercial code Abaqus/Explicit 2016-1 was used. The material behaviour of the steel and aluminium HP sections was described by an isotropic yield surface with associated flow and 
isotropic work hardening. The material models were calibrated from uniaxial tensile tests under quasi-static conditions. Uniaxial tensile tests for different material orientations yielded no significant anisotropic behaviour. The HP sections were discretized with reduced integrated shell elements of type S4R and five integration points in thickness direction. A mesh size of $2 \mathrm{~mm}$ was found to adequately describe the deformation behaviour. The behaviour of the adhesive was described by a bi-linear traction-separation law for all opening modes. The adhesive layer was discretized with cohesive elements of type COH3D8 and a mesh size of $2 \mathrm{~mm}$. They were connected to the shell elements of the HP sections by tied constraints. Contact between the top HP section, the impactor and the supports was modeled by a general contact algorithm with a friction coefficient of 0.2. Impactor and supports were modeled as rigid.

The Abaqus fastener formulation [5] was applied to model the macroscopic behaviour of SPR connections. Here, a connector element is placed in between the opposing surfaces. Nodes within a certain influence radius are connected through a distributing coupling. The macroscopic response of the connection is described by the connector behaviour. The formulation allows to define an elastic and plastic response, as well as damage and failure. The mode-mix ratio $\Psi_{m}$ represents an important quantity in describing the connector behaviour. It is defined by the ratio of the derived normal force $F_{N}$ and the derived shear force $F_{S}$.

$$
\Psi_{m}=\frac{2}{\pi} \tan ^{-1}\left(\frac{F_{N}}{F_{S}}\right)
$$

Consequently, the mode-mix ratio $\Psi_{m}$ takes on a value of 1 for pure normal loading and a value of 0 for pure shear loading. The derived components are calculated from the connector forces $f_{1}, f_{2}, f_{3}$ and moments $m_{1}, m_{2}, m_{3}$ (Figure 2).

$$
\begin{gathered}
F_{N}=\left|f_{3}\right|+\frac{K}{r} \sqrt{m_{1}^{2}+m_{2}^{2}} \\
F_{S}=\sqrt{f_{1}^{2}+f_{2}^{2}}
\end{gathered}
$$

The connector behaviour was calibrated by a reverse egi-
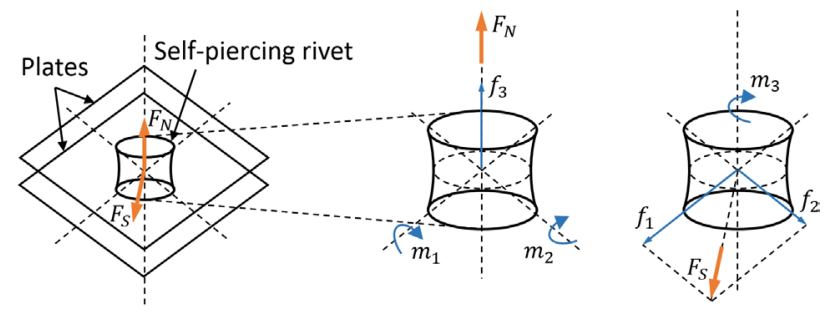

Fig. 2. Connector forces, moments and derived components after [5].

neering approach. Plates of the investigated material combination were joined by a single SPR connection to assemble a cross-shaped specimen. The specimens were then subjected to normal, shear and mixed-mode loading up to failure. The connector behaviour was calibrated based on the experimental force-displacement response (Figure 3).

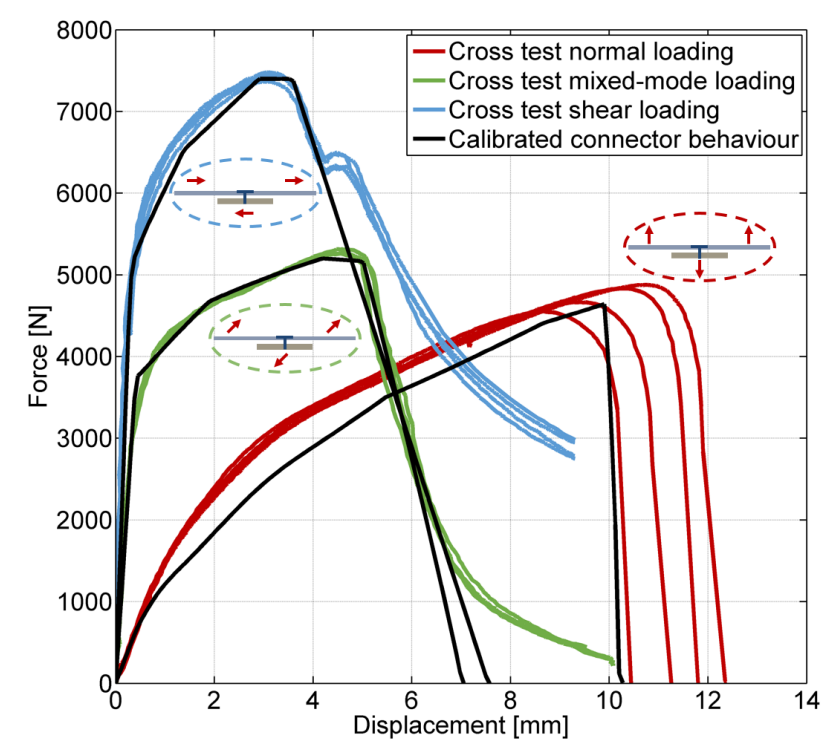

Fig. 3. Force-displacement response of the single SPR connection.

\subsection{Numerical results}

First simulation results showed that the open geometry of the HP sections implied unwanted deformations. The side walls of the HP sections started to bend inwards for increasing impactor displacement (Figure 4(a)). Therefore, support plates were bonded to the flanges of the HP sections (Figure 4(b)). The geometry of the HP sections

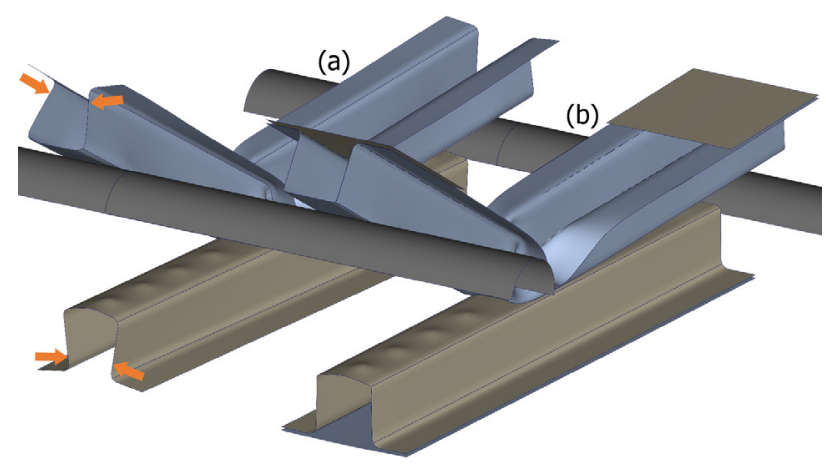

Fig. 4. Deformation behaviour of the HP sections without (a) and with support plates (b).

largely determines the loads introduced into the connections. It is vital to subject the connections to a wide range of different loading modes to enable a broad validation of the connection models. However, this can hardly be achieved with only one specimen configuration. Therefore, two configurations were developed, one for normal and one for shear dominated loading. In the normal configuration (Figure 5(a)), the top and bottom HP section have the same height. The top HP section is bent for increasing impactor displacement, while the bottom HP section remains straight. This results in a relative displacement normal to the connected surfaces. Hence, the connections are loaded in tension. In the shear configuration (Figure 5(b)), the height of the bottom HP section is 


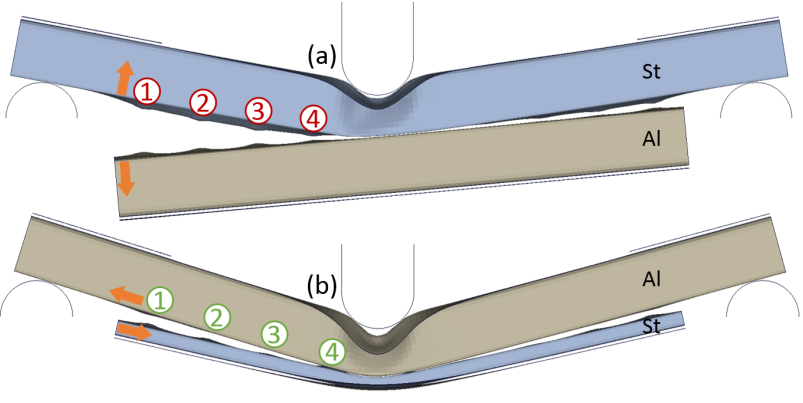

Fig. 5. Bending behaviour of the normal (a) and shear (b) configuration.

significantly reduced. Furthermore, the top HP section is now made from the $2.5 \mathrm{~mm}$ thick aluminium sheet and the bottom HP section is made from the $1.2 \mathrm{~mm}$ thick steel sheet. Both changes reduce the bending stiffness of the lower HP section significantly. The relative displacement is now tangential to the connected surface, resulting in a shear dominated loading. Slots were added to the top HP section in the shear configuration to avoid failure underneath the impactor (Figure 6). Failure in the connection

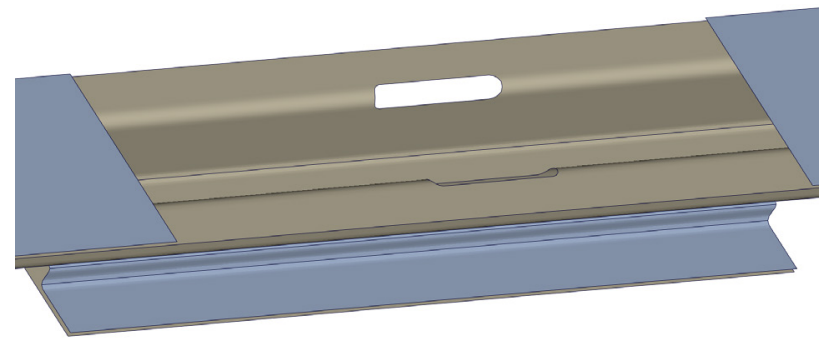

Fig. 6. Shear specimen configuration with slot in Top HP section.

was triggered from one side by an impactor offset. Successive failure of four SPR connections was predicted for both specimen configurations. The loading mode of SPR connections can be assed quantitatively by studying the mode-mix ratio $\Psi_{m}$ of the connector elements. The modemix ratio $\Psi_{m}$ was extracted at the onset of plastic deformation and connection failure (Figure 7). In the normal configuration, the mode-mix ratio $\Psi_{m}$ was mostly in the region above 0.5 . The loading mode changed from normal dominated mixed-mode loading to almost pure normal loading between the onset of plastic deformations and connection failure. In contrast, the mode-mix ratio $\Psi_{m}$ stayed below 0.4 in the shear configuration. Here, plastic deformations were initiated under almost pure shear loading. Connection failure occurred under shear dominated mixed-mode loading. Connection failure had a considerable impact on the force-displacement response (Figure 8). Failure of SPR connections resulted in a significant drop of the impactor force for both specimen configurations. In the normal configurations, failure of SPR connections occurred between an impactor displacement of $32 \mathrm{~mm}$ and $64 \mathrm{~mm}$. The failure sequence in the shear configuration started at a significantly higher impactor displacement and occurred more rapid. Here, the tangential relative dis-

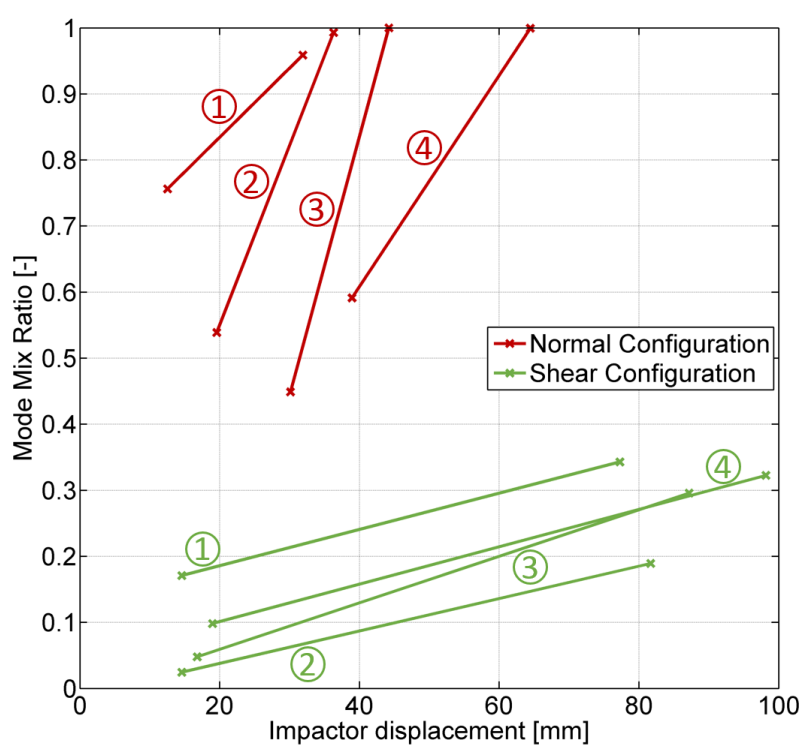

Fig. 7. Connector element mode-mix ratio $\Psi_{m}$ at onset of plastic deformations and failure.

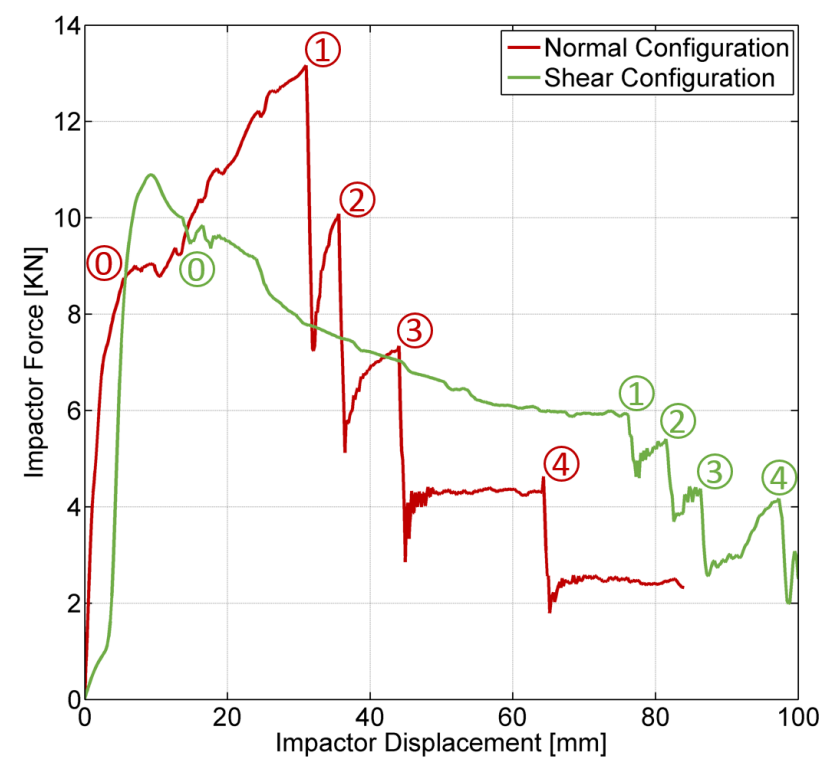

Fig. 8. Simulation force-displacement curves of the normal and shear configuration.

placement means that the load is distributed more evenly over the whole connection. Failure of the first cohesive element is marked by position 0 . Adhesive failure was initiated before failure of the first SPR connection for both configurations.

\section{Experimental analysis}

\subsection{Experimental setup}

Quasi-static experiments were perfomred on a electromechanical dual column testing machine. The normal and shear specimens were placed between a moveable impactor and fixed supports (Figure 9). Impactor and supports were both made from steel. All experiments were 


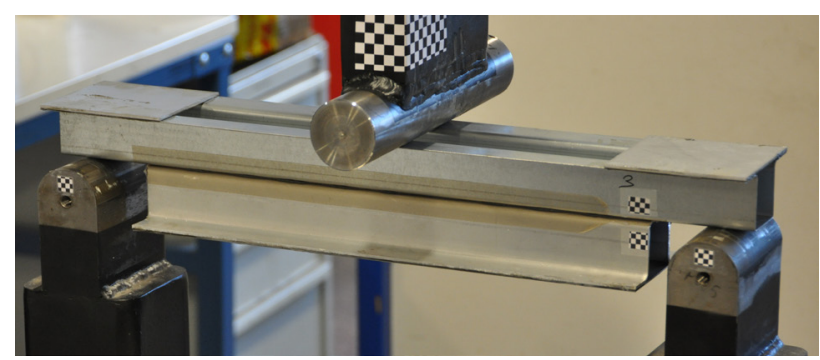

Fig. 9. 3-point bending test setup.

performed for a constant impactor velocity of $10 \mathrm{~mm} / \mathrm{min}$. The force at the impactor was recorded by a $250 \mathrm{kN}$ load cell. A camera system was used to track the impactor displacement and the relative movement of the HP sections. For that purpose, chessboard patterns were applied to the impactor and the specimens.

\subsection{Experimental results}

Figure 10 shows the impactor force-displacement curves for both specimen configurations. In the normal configu-

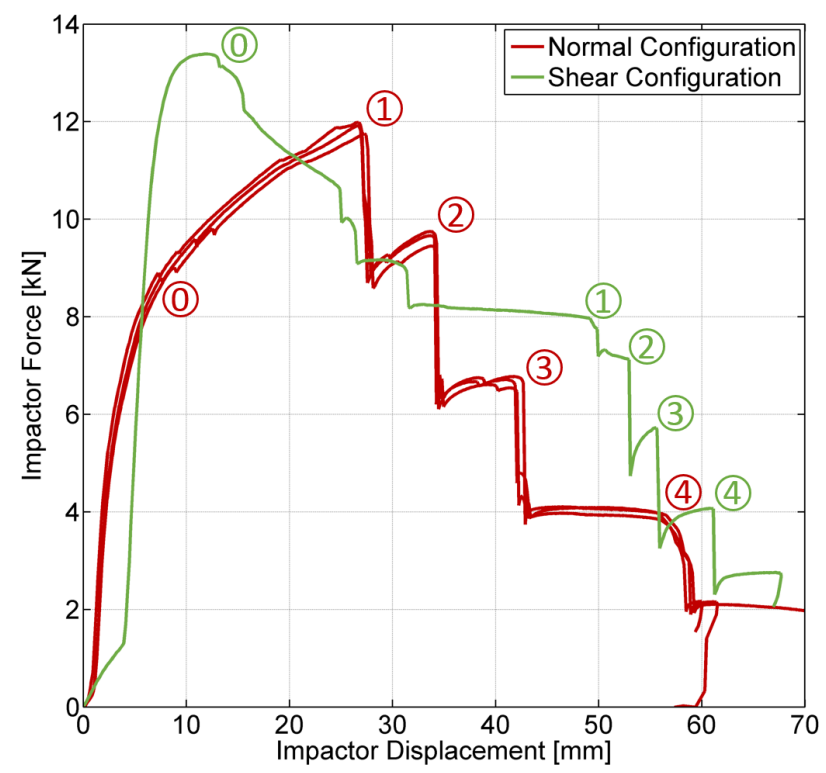

Fig. 10. Experimental force-displacement curves of the normal and shear configuration.

ration, forces increased linearly up to the point where the top HP section started to deform plastically underneath the impactor. At position 0, a crack was initiated inside the adhesive layer. The maximum force was reached right before failure of the first SPR connection at position 1 (Figure 11(a)). The crack inside the adhesive layer propagated towards the center of the specimen for increasing displacements accompanied by successive failure of three more SPR connections at positions 2 to 4 (Figure 11(b)).

The slot in the top HP section of the shear specimen had a distinct effect on the force-displacement response. Initially, the impactor was only in contact with the flanges

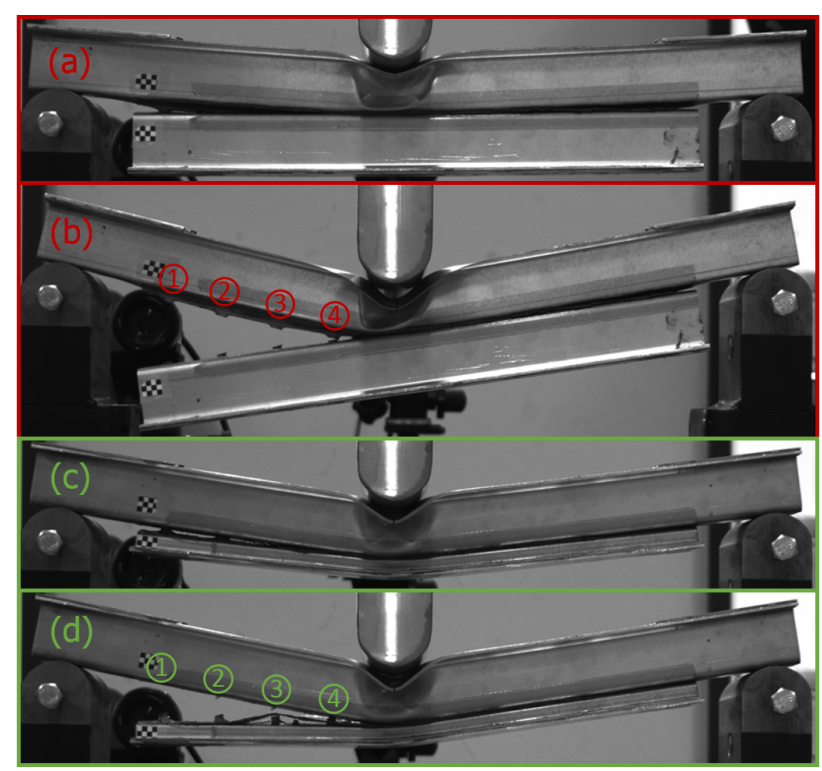

Fig. 11. Failure sequence of the normal (a),(b) and shear (c),(d) configuration.

before hitting the side walls of the top HP section after approximately $4 \mathrm{~mm}$ displacement. Forces increased rapidly up to the point where the top HP section started to buckle underneath the impactor. At position 0, a crack was initiated inside the adhesive layer. The crack propagated towards the center of the specimen for increasing impactor displacements. Figure 11(c) shows the shear specimen right before failure of the first SPR connection. In total, four SPR connections failed in short succession marked by positions 1 to 4 (Figure 11(d)).

The deformation behaviour of the HP sections is in good agreement with the numerical predictions. The bottom HP section remained straight in the normal configuration but bent significantly in the shear configuration, successfully introducing different loading modes into the connections. Consequently, the failure behaviour of the SPR connections differed significantly between both specimen configurations. In the normal configuration, the steel sheet was pulled over the rivet head and the rivet remained stuck in the aluminium sheet (Figure 12(a)). Local de-

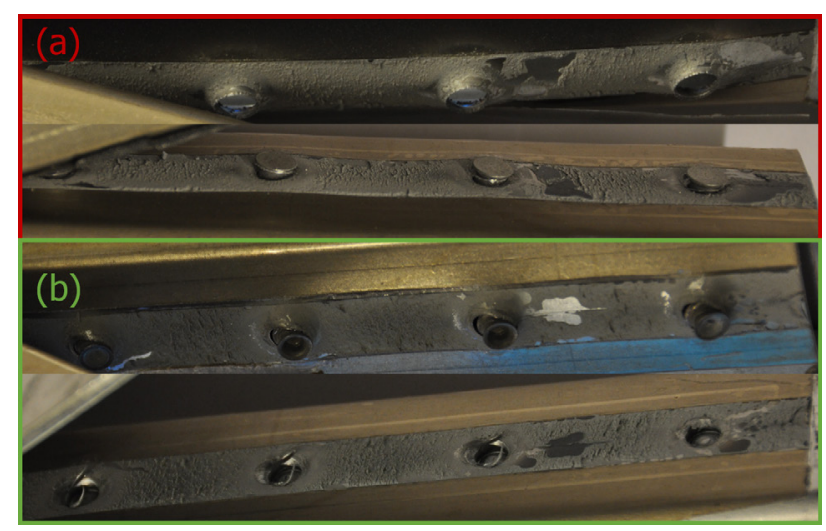

Fig. 12. Failure surfaces of the normal (a) and shear (b) configuration. 
formations in the steel sheet were symmetrical around the rivet axis, showing that the connection was loaded normal to the joint surface. In the shear configuration, the rivet was rotated and pulled out from the aluminium sheet (Figure 12(b)). The steel sheet was dragged underneath the rivet head on one side indicating a shear dominated loading. The observed failure behaviour of the SPR connections is in good agreement with the predicted connector mode-mix ratios $\Psi_{m}$ (Figure 7). The failure behaviour of the adhesive layer was dominated by cohesive failure for both configurations. However, small areas can be identified where no adhesive is present due to inclusions of air. These air inclusions were introduced during the riveting process.

\section{Conclusion}

A new component test to validate numerical models of steel-aluminium connections was presented. Test specimens consisted of two HP sections joined by adhesive bonding in combination with SPR. The specimens were tested in a 3-point bending setup up to failure of the adhesive and SPR connections. FE simulations were performed to optimize the loads introduced into the connections. As a result, two specimen configurations were proposed, one for normal dominated and one for shear dominated loading. Quasi-static experiments showed good agreement with the numerical predictions. Both specimen configurations yielded successive failure of multiple SPR connections with different failure modes. Cohesive failure of the adhesive connections was observed. The developed component test setup is therefore well suited for validating numerical models of hybrid connections. The setup will further be tested under impact loadings for validation of numerical connection models applied in vehicle crash analysis.

\section{References}

1. D. Morin, B. Bourel, B. Bennani, F. Lauro, D. Lesueur, International Journal of Impact Engineering 53, 94 - 105 (2013)

2. M. May, H. Voß, S. Hiermaier, International Journal of Adhesion and Adhesives 49, 7 - 17 (2014)

3. R. Porcaro, A. G. Hanssen, A. Aalberg, M. Langseth, International Journal of Crashworthiness 9, 141 - 154 (2004)

4. N. H. Hoang, A. G. Hanssen, M. Langseth, R. Porcaro, International Journal of Solids and Structures 49, 3211 3223 (2012)

5. Dassault Systemes, Abaqus 2016 Documentation (2016) 
